\title{
Infectivity and viability of dengue virus infected hepatocytes cocultured with peripheral blood mononuclear cells from a healthy subject
}

\author{
Sekar Asri Tresnaningtyas, ${ }^{1}$ Fithriyah Sjatha, ${ }^{2,3}$ Beti Ernawati Dewi ${ }^{2,3}$
}

pISSN: 0853-1773 - elSSN: 2252-8083 https://doi.org/10.13181/mji.oa.203433 Med J Indones. 2020;29:260-7

Received: February 06, 2019 Accepted: June 27, 2020

Authors' affiliations:

${ }^{1}$ Master Program in Biomedical Sciences, Faculty of Medicine, Universitas Indonesia, Jakarta, Indonesia, ${ }^{2}$ Department of Microbiology, Faculty of Medicine, Universitas Indonesia, Cipto Mangunkusumo Hospital, Jakarta, Indonesia, ${ }^{3}$ Infectious Disease and Immunology Research Center, Indonesian Medical Education and Research Institute, Jakarta, Indonesia

\section{Corresponding author:}

Beti Ernawati Dewi

Department of Microbiology, Faculty of Medicine, Universitas Indonesia, Jalan Pegangsaan Timur No. 16, Pegangsaan, Menteng, Central Jakarta 10320, DKI

Jakarta, Indonesia

Tel/Fax: +62-21-3160491

E-mail: betied@yahoo.com

\begin{abstract}
BACKGROUND Dengue virus (DENV) can infect and replicate in monocytes, resulting in antibody-dependent enhancement. The liver is the main target of DENV, and the infection mechanisms of DENV include direct cytopathic effects (CPEs) of the virus, mitochondrial dysfunction, and effect of cellular and humoral immune factors in the liver. This study was aimed to explore the infectivity of DENV and viability of human hepatocytes using Huh 7it-1 cells cocultured with peripheral blood mononuclear cells (PBMCS).
\end{abstract}

METHODS Huh 7it-1 cells were infected with dengue virus serotype-2 (DENV-2) New Guinea C strain at multiplicity of infection of 0.5 and $1 \mathrm{FFU} / \mathrm{cell}$, and cocultured in vitro with and without adherent PBMCs. The infectivity of DENV was assessed by immunoperoxidase staining. The viability of Huh 7it-1 cells was assessed using 3-(4,5-dimethylthiazol-2-yl)-2,5-diphenyltetrazolium bromide (MTT, a tetrazole) assay and trypan blue staining. Data were statistically analyzed by Shapiro-Wilk and analysis of variance for normality significances.

RESULTS The result showed that addition of PBMCS to DENV-2 infected Huh 7it-1 cells decreased the infectivity of DENV (15-37\%). DENV-2 infection decreased the viability of Huh 7it-1 cells (15.5-20.8\%). Despite the decrease in infectivity of DENV, the addition of PBMCs increased the Huh 7it-1 cells viability (4.5-10.2\%).

CONCLUSIONS Addition of PBMCs to Huh 7it-1 cells that are infected with DENV-2 decreased the infectivity of DENV and increased Huh 7it-1 cells viability.

KEYWORDS dengue, hepatocytes, in vitro, liver, monocytes
Dengue virus (DENV) is a mosquito-borne virus transmitted by vectors Aedes aegypti and $A$. albopictus. DENV infection is widespread in all tropical and subtropical regions. ${ }^{1}$ World Health Organization estimated that the incidence of DENV infection reaches 50-100 million cases in the world every year, and the incidence of DENV infection has increased 30fold over the last 50 years. ${ }^{2}$ Based on the prevalence of dengue studies, 3.9 billion people in 128 countries in the world have a risk of being infected with DENV. ${ }^{3}$
Based on an in vivo study, it was observed that DENV can infect monocytes, ${ }^{4}$ platelets, ${ }^{5}$ endothelial cells, ${ }^{6}$ hepatocytes, ${ }^{7}$ and Kupffer cells. ${ }^{8}$ Monocytes and macrophages are thought to be cells that can support DENV replication and lead to antibodydependent enhancement hypothesis, which explains the risk of severity of DENV infection caused by a secondary infection with heterologous serotypes. ${ }^{1}$ In an in vitro study, it was seen that DENV can infect various hepatocyte strains, including Huh7 cells, 
primary liver carcinoma (PLC), Hep3B, Chang liver cells, and HA22T. ${ }^{9}$

Some mechanisms involved in the damage of hepatocytes during DENV infection are direct and indirect cytopathic effects (CPEs). The direct CPE is a damage due to viral infection, while indirect CPE is due to hypoxia and the influence of cellular and humoral immune factors in the liver. ${ }^{10}$ The pathogenesis of DENV related to liver cell damage is still not clearly known. To determine the role of DENV and monocytes in liver cell damage, we studied DENV infection in Huh 7it-1 hepatocyte cell lines cultured in vitro with peripheral blood mononuclear cells (PBMCs) and assessed the infectivity and cell viability.

\section{METHODS}

This research was conducted from February to December 2018 in the laboratory of virology and molecular biology, Department of Microbiology, Faculty of Medicine, Universitas Indonesia. This study used PBMCs from a healthy subject, Huh 7it-1 liver cancer cells (obtained from Dr. Chie Aoki at Kobe University), and dengue virus serotype-2 New Guinea C (DENV-2 NGC) strain from the collections of the laboratory of virology and molecular biology, Department of Microbiology, Faculty of Medicine, Universitas Indonesia. This research has ethical permission from the Ethics Committee of the Faculty

Table 1. The 12 treatment groups of this study

\begin{tabular}{lcc}
\hline $\begin{array}{l}\text { Treatment } \\
\text { group* }\end{array}$ & DENV-2 & PBMCs \\
\hline I & MOI 0.5 FFU/cell & V \\
II & MOI 0.5 FFU/cell & - \\
III & MOI 1 FFU/cell & V \\
IV & MOI 1 FFU/cell & - \\
V & UV-inactivated DENV-2 & V \\
VI & UV-inactivated DENV-2 & - \\
VII & Heat-inactivated DENV-2 & V \\
VIII & Heat-inactivated DENV-2 & - \\
IX & Supernatant of Huh 7it-1 cell culture & V \\
X & Supernatant of Huh 7it-1 cell culture & - \\
XI & Huh 7it-1 cell medium & V \\
XII & Huh 7it-1 cell medium & - \\
\hline
\end{tabular}

DENV-2=dengue virus serotype-2; PBMCs=peripheral blood mononuclear cells; $\mathrm{MOI}=$ multiplicity of infection; FFU=focus-forming units; UV=ultraviolet

*All treatment groups were cultured in Huh 7it-1 cell of Medicine, Universitas Indonesia (No. 248/UN2.F1/ ETIK/2018). In this study, we arranged 12 treatment groups as shown in Table 1.

This study started with infecting Huh 7it-1 cells using DENV-2 NGC strain at multiplicity of infection (MOI) of 0.5 and 1 focus-forming units (FFU)/cell (Table 1). After 2 hours of incubation, the treatment was continued with in vitro coculturing with and without adherent PBMCs for 72 hours. The infectivity of DENV was assessed through immunoperoxidase staining. The viability of Huh 7it-1 cells was assessed using 3-(4,5-dimethylthiazol-2-yl)-2,5-diphenyltetrazolium bromide (MTT, a tetrazole) assay and trypan blue staining. The workflow diagram is shown in Figure 1.

\section{Propagation of Huh 7it-1 cells}

The frozen Huh 7it-1 cell stock in the cryotube was thawed and quickly resuspended with Dulbecco's modified Eagle medium (DMEM) (Gibco, USA), and then centrifuged at 1,200 rpm for $4 \mathrm{~min}$. The pellet was resuspended with $5 \mathrm{ml}$ DMEM 10\% FBS (fetal bovine serum) (Gibco) and replaced to a T25 flask followed by incubation at $37^{\circ} \mathrm{C}$ with $5 \% \mathrm{CO}_{2}$. The medium was replaced daily until a monolayer had formed on the flask surface (observed with an inverted microscope). Cell passaging was carried out by removing the medium in the flask and washing it with $2 \mathrm{ml}$ phosphate buffered saline (PBS) $1 \times$, followed by adding $500 \mu \mathrm{l}$ trypsin 0.25 ethylenediaminetetraacetic acid (Gibco). The flask was then incubated at $37^{\circ} \mathrm{C}$ with $5 \% \mathrm{CO}_{2}$ for 3-5 min until the cells were detached from the surface of the flask (observed with an inverted microscope). Next, the flask was filled with $4 \mathrm{ml}$ of DMEM without

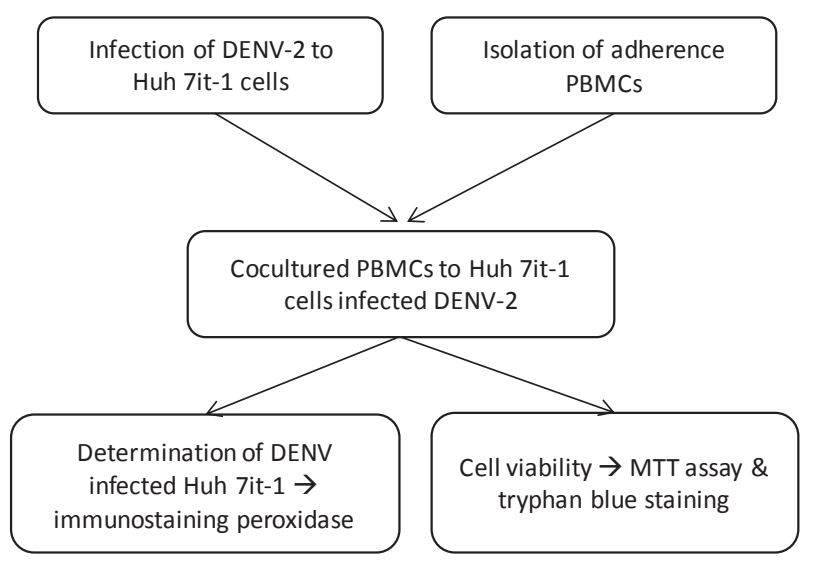

Figure 1. Workflow diagram. DENV-2=dengue virus serotype-2; PBMCs=peripheral blood mononuclear cells; DENV=dengue virus; MTT=3-(4,5-dimethylthiazol-2-yl)-2,5diphenyltetrazolium bromide 
FBS, resuspended and transferred into a tube, and centrifuged at a speed of 1,200 rpm for 4 min. Pellets were resuspended in the DMEM $10 \% \mathrm{FBS}$ and then kept in several flasks. These flasks were then incubated at $37^{\circ} \mathrm{C}$ with $5 \% \mathrm{CO}_{2}$ until confluence.

\section{Propagation of DENV-2 NGC strain}

DENV-2 NGC strains were propagated in Vero cells in a T75 flask. Medium of the monolayer Vero cells was infected with $500-1,000 \mu \mathrm{l}$ of DENV-2 NGC strains. After 2 hours of incubation, $10 \mathrm{ml}$ MEM 2\% FBS was added to the flask and incubated at $37^{\circ} \mathrm{C}$ with $5 \% \mathrm{CO}_{2}$. The harvesting of the supernatant (which contains the DENV-2) was carried out on the 5-6th day when CPE appeared. Virus titers were measured by immunoperoxidase staining and stored in a $1 \mathrm{ml}$ tube at $-80^{\circ} \mathrm{C}$ until used.

\section{Isolation of PBMCs}

In this study, we used $20 \mathrm{ml}$ of whole blood from a healthy subject who was vaccinated with Japanese encephalitis virus vaccine. Serum from whole blood was tested for IgG, IgM, and dengue NS1. The isolation of PBMCs from whole blood were done according to the previous study. ${ }^{11}$ Five milliliters of whole blood was suspended with $2 \mathrm{ml} \mathrm{PBS}$ in a tube and transferred slowly through the tube wall into a falcon tube, which contained $5 \mathrm{ml}$ of Ficoll-Paque (GE Healthcare, USA). Then, it was centrifuged at 2,500 rpm for $30 \mathrm{~min}$ with swing rotor and brake off. The PBMC rings were collected in a tube and washed with Roswell Park Memorial Institute (RPMI) 1640 medium (Sigma, USA) without FBS, and then centrifuged at 1,200 rpm for $5 \mathrm{~min}$.

Adherent PBMCs were isolated as described in the previous study. ${ }^{12}$ The pellets were resuspended in $5 \mathrm{ml} \mathrm{RPMI} \mathrm{10 \%} \mathrm{FBS} \mathrm{with} 1 \%$ Penicillin-Streptomycin (Gibco). The suspension was placed on a T25 flask and incubated at $37^{\circ} \mathrm{C}$ with $5 \% \mathrm{CO}_{2}$ incubator for 2 hours. Non-adherent PBMCs that were not attached to the flask were removed, and the flask was washed twice with RPMI medium without FBS. The cells attached in the flask were pipetted to obtain adherent PBMCs. The suspension contained adherent PBMCs centrifuged at a speed of 1,200 rpm for 5 min. Pellets were resuspended in RPMI 10\% FBS. The calculation of the number of adherent PBMCs was undertaken using hemocytometer, with the cell concentration of $1 \times 10^{6}$ cells $/ \mathrm{ml}$.

\section{Infecting Huh 7it-1 cells with DENV 2}

Huh 7it-1 cells were grown on 96 microwell plates with a concentration of $5 \times 10^{5} \mathrm{cells} / \mathrm{ml}$. The microwell plates were incubated for 24 hours at $37^{\circ} \mathrm{C}$ with $5 \%$ $\mathrm{CO}_{2}$ until a monolayer was formed, then the medium was discarded from the well. Cells on the microwell plates were infected with $50 \mu \mathrm{LENV}-2$ at various titers according to the treatments including UV- and heatinactivated DENV-2, supernatant cell, and medium as negative control. The microwell plates were incubated for 2 hours at $37^{\circ} \mathrm{C}$ with $5 \% \mathrm{CO}_{2}$ and agitated every 30 min.

\section{Coculturing of DENV-2-infected Huh 7it-1 cells with PBMCs}

After 2 hours of incubation, $50 \mu \mathrm{l}$ of adherent PBMCs with a concentration of $1 \times 10^{6}$ cells $/ \mathrm{ml}$ was added into each well, meanwhile, RPMI 1640 medium (Sigma) was added in the control treatment. The microwell plates were then incubated at $37^{\circ} \mathrm{C}$ with $5 \%$ $\mathrm{CO}_{2}$ for 72 hours.

\section{Observation of cell viability with trypan blue staining}

Cell viability was observed through trypan blue staining by adding $50 \mu \mathrm{l} /$ well of trypan blue dye. Cells on the microwell plates were observed using an inverted microscope with $100 \times$ magnification. The colored cells were dead cells and transparent cells were living cells.

\section{Calculation of cell viability using MTT assay}

Cell viability was also observed using MTT assay by adding $150 \mu \mathrm{l}$ of $10 \%$ MTT solution into each well in microwell plates. Microwell plates were then incubated at $37^{\circ} \mathrm{C}$ with $5 \% \mathrm{CO}_{2}$ incubator for 3 hours. The solution in microwell plates was then discarded, and $100 \mu \mathrm{l}$ of $100 \%$ dimethyl sulfoxide was added to every well. MTT result was read using an enzymelinked immunosorbent assay (ELISA) reader at a wavelength of $490 \mathrm{~nm}$. In this study, the viability of Huh 7it-1 cells infected with DENV-2 in various treatments after 72 hours of incubation was assessed using MTT assay and trypan blue staining. MTT assay result was obtained in terms of absorbance value/ optical density (OD). The percentage of cell viability was obtained by comparing the OD of each treatment with that of the negative control treatment (Huh 7it-1 cells which treated with the addition of Huh 7it-1 cell medium). 


\section{Determination of DENV-infected Huh 7it-1 cells}

The number of DENV-infected cells was measured by immunostaining assay according to previous study with a slight modification by replacing the treatment using methyl cellulose with Huh 7 it-1 cells for 72 hours. ${ }^{13}$ The solution in the microwell plate was discarded after 72 hours of treatment. Cells were fixated by adding $200 \mu \mathrm{l}$ of formalin $3.7 \%$ in PBS $1 \times$ into each well, which were then incubated for $15 \mathrm{~min}$ at room temperature and washed three times using PBS $1 \times$ with each washing followed by 5 min of incubation. Next, 100 $\mu$ l of Triton X $0.5 \%$ (Sigma) in PBS was added into each well in the microwell plate, incubated for $10 \mathrm{~min}$ at room temperature, and washed with $200 \mu$ PBS 1x three times without incubation. Cells were added with $50 \mu \mathrm{l}$ of primary antibodies (human IgG anti-DENV) in skim milk blocking buffer of 1/500 dilution, continued with incubation for 1 hour at room temperature. After 1 hour of incubation, microwell plates were washed with $200 \mu$ PBS 1x. Washing was done three times, with each washing followed by 5 min of incubation. The process was continued by adding $50 \mu \mathrm{l}$ of secondary antibody (Goat Anti-Human IgG-labeled HRP [Invitrogen, USA]) in skim milk blocking buffer (Sigma) 1/500 dilution into each well and washed as previously done. Then, $100 \mu$ l of 3,3'-diaminobenzidine substrate $1 \times$ was added, incubated for $15 \mathrm{~min}$ at room temperature, and washed as previously done. Furthermore, stained cells were observed with an inverted microscope and calculated to determine the number/percentage of the infected cells.

\section{Statistical analysis}

Data were analyzed using SPSS version 23 (IBM Corp., USA). Normality of the data was analyzed with Shapiro-Wilk test. The significance of differences of each treatment was analyzed by analysis of variance compared with control treatment. The differences were considered significant when the $p$-value $<0.05$.

\section{RESULTS}

\section{Effect of DENV-2 infection and coculturing with PBMCs on infectivity}

The results showed that Huh 7it-1 cells which were treated with DENV-2 at MOI of 1 FFU/cell (95\%) was greater than the number of cells infected with DENV-2 at an MOI of $0.5 \mathrm{FFU} /$ cell (57\%) (Figure 2, a and $b$ ). Whereas there was no infection had occurred in control treatments, such as Huh 7it-1 cells, which were treated with supernatant of Huh 7it-1 cell culture (Figure 2c), UV-inactivated DENV-2 (Figure 2d), heatinactivated DENV-2 (Figure 2e), and Huh 7it-1 cell medium (Figure $2 \mathrm{f}$ ). It was observed that no cells were stained (brown color). However, the infectivity of Huh 7it-1 cells in the treatment group that was treated with cocultured PBMCs showed different results compared to that of the group without PBMCs. The number of infected cells from DENV-2 infection at an MOI of $1 \mathrm{FFU} /$ cell without PBMCs (Figure 2b) was 95\% and that from DENV-2 infection at an $\mathrm{MOI}$ of $1 \mathrm{FFU} /$ cell cocultured with PBMCs (Figure $2 \mathrm{~g}$ ) was $80 \%$. The same result was found in the treatment of infection from DENV-2 at an MOI of $0.5 \mathrm{FFU} /$ cell; number of infected cells were $57 \%$ (without PBMCs) and 20\% (cocultured with PBMCs) (Figure 2, $a$ and h).

\section{Effect of DENV-2 infection and coculturing with PBMCs on cell viability}

The percentage of viability of Huh 7it-1 cells treated with UV-inactivated DENV-2, heat-inactivated DENV2 , and Huh 7it-1 cell medium were significantly higher ( $p=0.001$ ) compared to that of Huh 7it-1 cells that were infected with DENV-2 at MOI of 0.5 and $1 \mathrm{FFU} /$ cell and supernatant of Huh 7it-1 cell culture as shown in Table 2. The result also showed that the viability percentage of Huh 7it-1 cells that were infected with DENV-2 at an $\mathrm{MOI}$ of $0.5 \mathrm{FFU} /$ cell was significantly higher $(p=0.014)$ than those infected with DENV-2 at an MOI of 1 FFU/ cell. The cell viability in treatment groups of Huh 7it-1 cells cocultured with PBMCs had the same results. The viability percentage of Huh 7it-1 cells treated with UVinactivated DENV-2, heat-inactivated DENV-2, and Huh 7it-1 cell medium were significantly higher $(p<0.001)$ compared to that of Huh 7it-1 cell infected with DENV2 at MOI of 0.5 and $1 \mathrm{FFU} /$ cell and supernatant of Huh 7it-1 cell culture.

The viability percentage of Huh 7it-1 cells infected with DENV-2 at an MOI of $0.5 \mathrm{FFU} /$ cell cocultured with PBMCs was not significantly higher $(p=0.068)$ compared to those treated without PBMCs. A different result was found in the treatment of Huh 7it-1 cells infected with DENV-2 at an MOI of $1 \mathrm{FFU} /$ cell. In this case, the viability percentage of Huh 7it-1 cells cocultured with PBMCs was significantly different $(p<0.001)$ compared to those treated without PBMCs. In the treatment with and without cocultured PBMCs, the viability percentage of Huh 7it-1 cells which were 

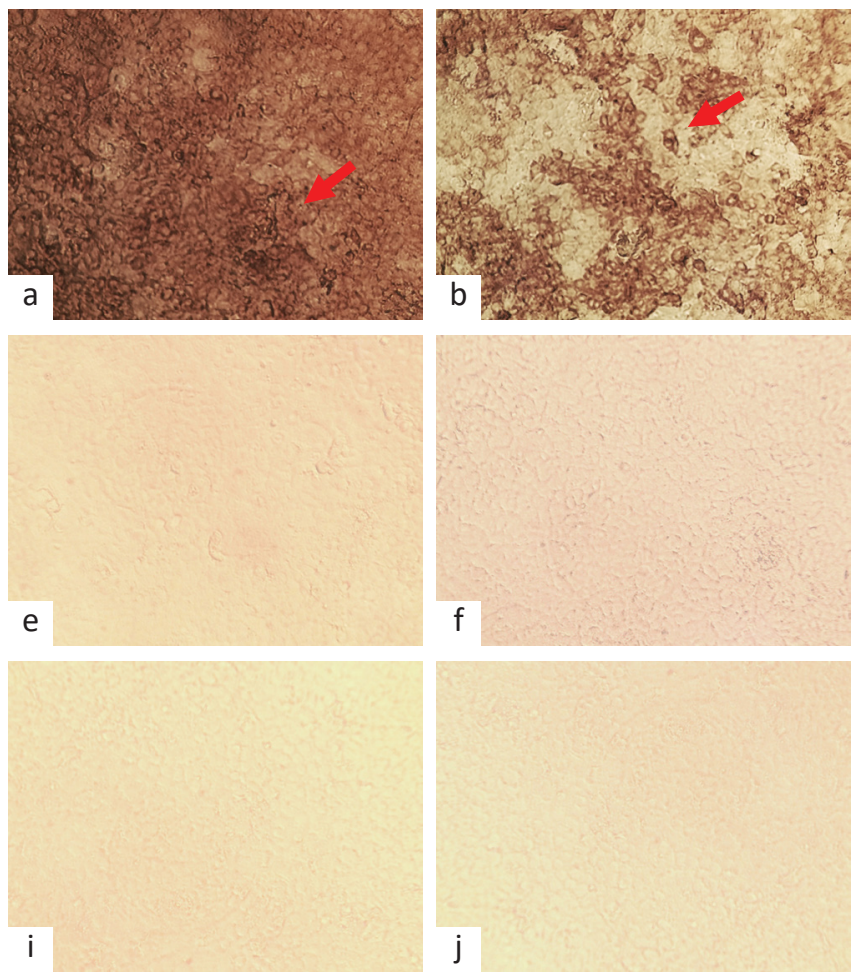
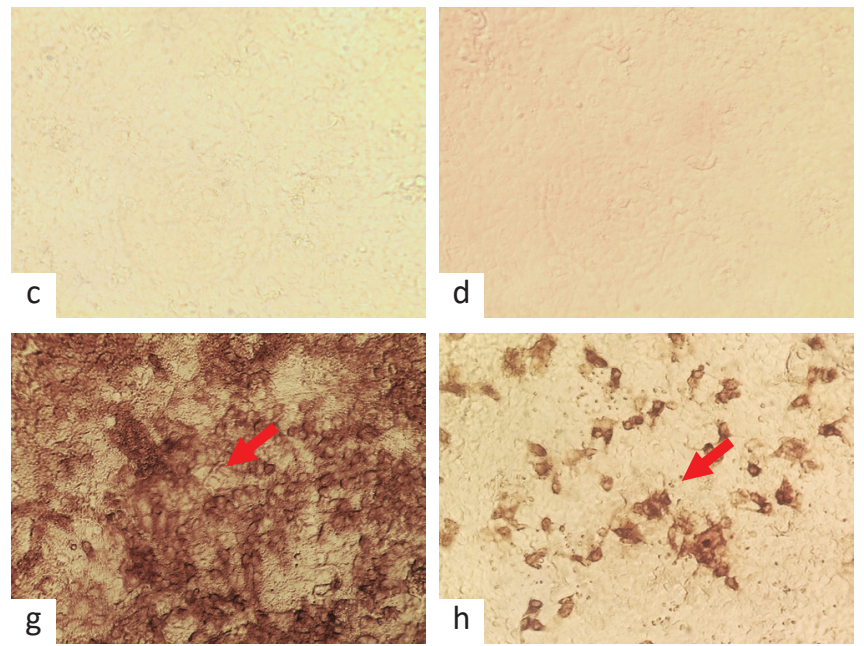

g
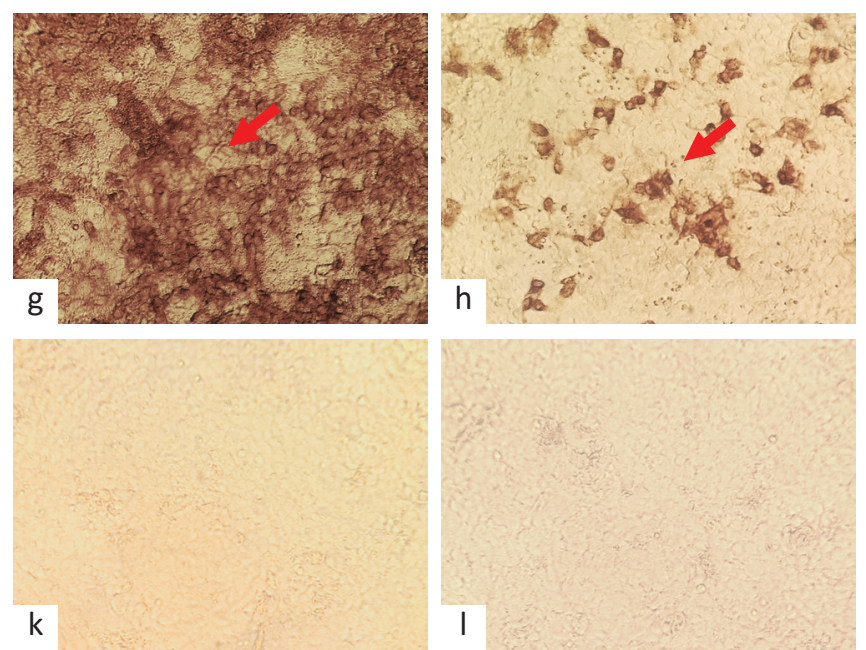

Figure 2. Microscopic view (200x magnification) of immunostaining results of Huh 7it-1 cells treated with (a) DENV-2 at an MOI of $1 \mathrm{FFU} /$ cell without PBMCs (red arrow); (b) DENV-2 at an MOI of $0.5 \mathrm{FFU} /$ cell without PBMCs (red arrow); (c) supernatant of Huh 7it-1 cell culture without PBMCs; (d) UV-inactivated DENV-2 without PBMCs; (e) heat-inactivated DENV-2 without PBMCs; (f) Huh 7 it-1 cell medium without PBMCs; (g) DENV-2 at an MOI of 1 FFU/cell cocultured with PBMCs (red arrow); (h) DENV-2 at an MOI of 0.5 FFU/cell cocultured with PBMCs (red arrow); (i) UV-inactivated DENV-2 cocultured with PBMCs; (j) heat-inactivated DENV-2 cocultured with PBMCs; (k) supernatant of Huh 7it-1 cell culture cocultured with PBMCs; and (I) Huh 7it-1 cell medium cocultured with PBMCs. DENV-2=dengue virus serotype-2; MOI=multiplicity of infection; FFU=focus-forming units; PBMCs=peripheral blood mononuclear cells; UV=ultraviolet

Table 2. Percentage of cell viability and infectivity

\begin{tabular}{lcccc}
\hline \multirow{2}{*}{ Treatment } & \multicolumn{2}{c}{ Cell viability* (\%) } & \multicolumn{2}{c}{ Infectivity $^{\dagger}(\%)$} \\
\cline { 2 - 5 } & Without PBMCs & Cocultured with PBMC & Without PBMCs & Cocultured with PBMCs \\
\hline Huh 7it-1 cell medium (control) & 100 & 100 & 0 & 0 \\
UV-inactivated DENV-2 & 101.55 & 102.97 & 0 & 0 \\
Heat-inactivated DENV-2 & 97.60 & 99.87 & 0 & 0 \\
Supernatant of Huh 7it-1 cell culture & $83.94^{\ddagger}$ & $88.70^{\ddagger}$ & 0 & 0 \\
DENV-2 MOI 0.5 FFU/cell & $84.48^{\ddagger}$ & $88.95^{\ddagger}$ & $57^{\ddagger}$ & $20^{\ddagger}$ \\
DENV-2 MOI 1 FFU/cell & $79.17^{\ddagger}$ & $89.44^{\ddagger}$ & $95^{\ddagger}$ & $80^{\ddagger}$ \\
\hline
\end{tabular}

PBMCs=peripheral blood mononuclear cells; UV=ultraviolet; DENV-2=dengue virus serotype-2; MOI=multiplicity of infection; FFU=focus-forming units *Result of MMT assay; ${ }^{\dagger}$ result of immunoperoxidase; "the value is significantly different $(p<0.05)$ compared to control treatment

not infected with DENV-2 was significantly higher $(p=$ $<0.001-0.03$ ) than the treatments which involved cells infected with DENV-2.

The cell viability result from trypan blue staining showed that the number of dead cells after the treatment of Huh 7it-1 cells were infected with DENV2 at an MOl of 0.5 (Figure 3a) and 1 FFU/cell (Figure 3b) was higher compared to when Huh 7it-1 cells with supernatant of Huh 7it-1 cell culture (Figure 3c), UVinactivated DENV-2 (Figure 3d), heat-inactivated DENV2 (Figure 3e), and Huh 7it-1 cell medium (Figure 3f). The difference between the staining results of Huh 7it-1 cells when cocultured with PBMCs and without PBMCs was not clearly visible. 

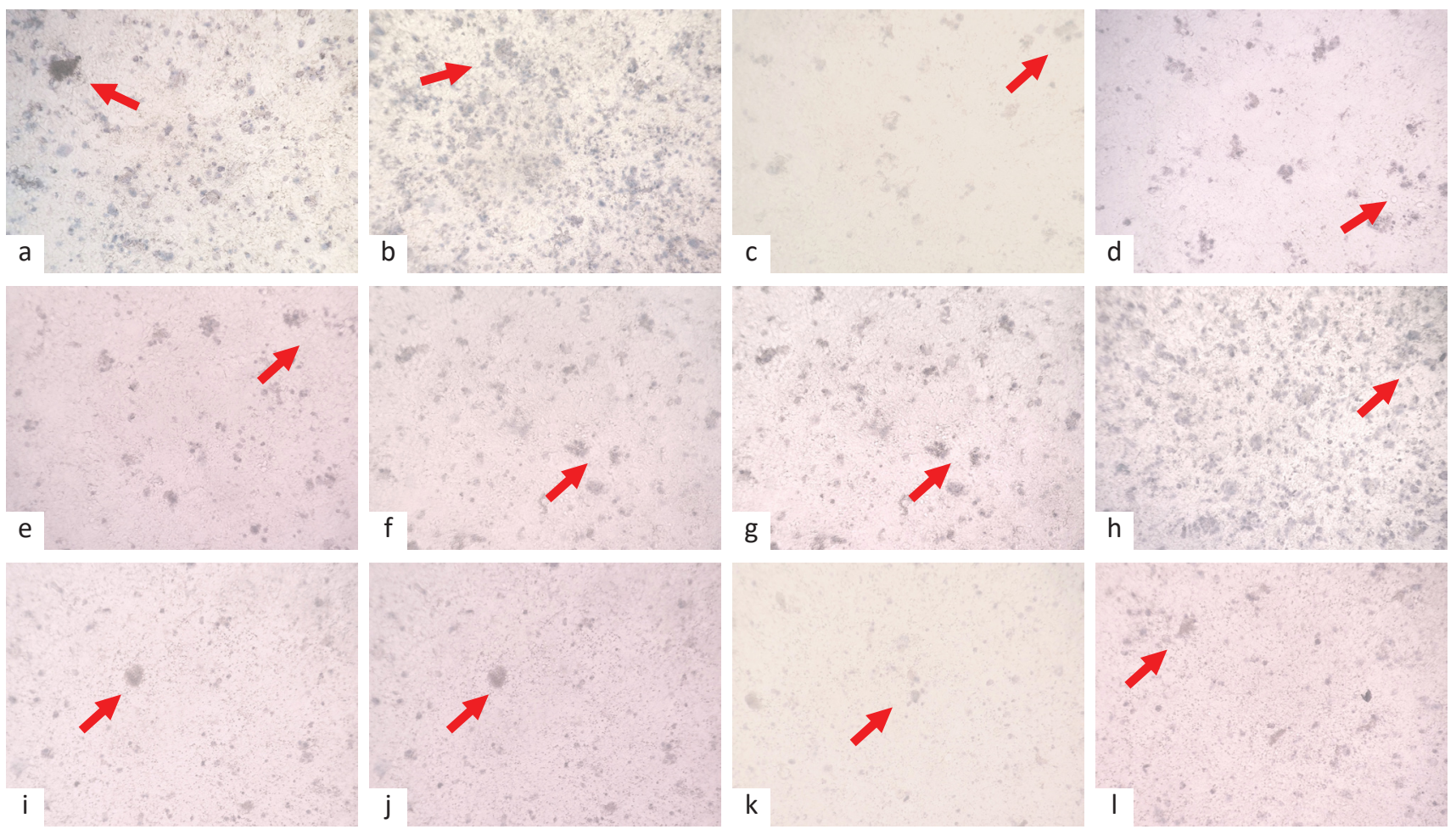

Figure 3. Microscopic view (100x magnification, pointed with red arrow) of trypan blue staining results of Huh 7it-1 cells treated with (a) DENV-2 at an MOI of 0.5 FFU/cell cocultured with PBMCs; (b) DENV-2 at an MOI of 1 FFU/cell cocultured with PBMCs; (c) supernatant of Huh 7it-1 cell culture without PBMCs; (d) UV-inactivated DENV-2 cocultured with PBMCs; (e) heat-inactivated DENV-2 cocultured with PBMCs; (f) Huh 7it-1 cell medium without PBMCs; (g) DENV-2 at an MOI of 0.5 FFU/cell without PBMCs; (h) DENV-2 at an MOI of 1 FFU/cell without PBMCs; (i) UV-inactivated DENV-2 without PBMCs; (j) heat-inactivated DENV-2 without PBMCs; (k) supernatant of Huh 7it-1 cell culture cocultured with PBMCs; and (I) Huh 7it-1 cell medium cocultured with PBMCs. DENV-2=dengue virus serotype-2; $\mathrm{MOI}=$ multiplicity of infection; FFU=focus-forming units; PBMCs=peripheral blood mononuclear cells; UV=ultraviolet

\section{DISCUSSION}

The severity of certain DENV infections can cause interference with the liver, such as hepatomegaly, and even lead to liver dysfunction. In severe DENV infection cases, liver injury due to hepatocyte apoptosis can be observed. ${ }^{14}$ In this study, we have used Huh 7 it-1 line cells, which are human hepatocyte line cells, to resemble the actual infection state. The results showed that there were differences between morphology of Huh 7it-1 cells infected with DENV-2 and those not infected with DENV-2. DENV is known to infect and replicate in Huh 7it-1 cells; therefore these cells are often used as an in vitro model for the DENV research. ${ }^{9}$ The effect on the infectivity of DENV-2-infected cells cocultured with PBMCs were assessed using immunostaining assay after 72 hours of incubation. The infected cells appeared brown as shown in Figure 2. In this study, we also found that DENV-2 infections with higher MOI values had increased infectivity compared to DENV-2 infections with lower MOI values. Hence, it was proved that high titers could infect more Huh 7it-1 cells than low DENV-2 titers. DENV can infect hepatocytes including Huh 7it-1 cells, by attaching the protein $\mathrm{E}$ virion to receptors on host cells, such as heparan sulfate, ${ }^{15}$ glucose-regulated protein $78,{ }^{16}$ and laminin, which are commonly found in the majority of liver cell lines. ${ }^{17}$ The pre-membrane structural protein in DENV-2 can bind to the claudin-1 receptor in Huh 7it-1 cells. Thus, DENV-2 can attach to the claudin-1 receptor and facilitate the entry of the virus into the cell. DENV2 can also infect other cell lines, such as PLC, Hep3B, Chang liver cells, and HA22T. Experiments by Lin et al9 showed that Huh7 cells, PLC, Hep3B, and Chang liver cells have higher replication rates, virion production levels, and aspartate aminotransferase levels.

PBMCs are part of blood cells consisting of lymphocytes ( $T$ cells, B cells, and natural killer cells), monocytes, and dendritic cells. PBMCs can secrete cytokines. We have used adherent PBMCs, which attached to the surface of the flask when incubated for approximately 2 hours. PBMCs that attached to the 
plate are known as monocytes. In a previous study, it was also stated that adherent PBMCs were able to reduce the transendothelial electrical resistance in endothelial cells, where it was suspected that adherent PBMCs were able to secrete more cytokines compared to non-adherent PBMCs. ${ }^{11}$ In addition, adherent PBMCs are also known as cells that support the replication of DENV in vivo. ${ }^{18}$ Cellular receptors reported to be used by DENV to enter cells are dendritic cell-specific intercellular adhesion molecule3-grabbing nonintegrin, which is owned by monocytes derived from dendritic cells, ${ }^{19}$ and Fc receptors in cases of secondary infection. ${ }^{20}$

In this study, the result of trypan blue staining showed that dead cells are colored by trypan blue or appear to be blue (showed by the red arrow in Figure 3), while living cells are not stained or appear to be transparent. It was also observed that the addition of PBMCs to Huh 7it-1 cells infected with DENV-2 resulted in decreased virus infectivity. This decrease in infectivity was caused by the mechanism of protection by interferons (IFNs), which are produced by monocytes in PBMCs. DENV can infect and replicate in monocytes. One of the host cell defense mechanisms against viral infections is IFN production. ${ }^{21}$ The results of research conducted by Kurane et $\mathrm{al}^{22}$ showed that IFN activity was detected in monocytes 12 hours after DENV infection and it reached the highest levels in 72 hours. Monocytes are thought to regulate $\mathrm{T}$ - and B-cell responses specific to DENV through antigen presentation and interleukin (IL)-1 production. In addition, monocytes also produce and induce IFN. ${ }^{19}$

This study showed that DENV-2 infection caused a decrease in Huh 7it-1 cell viability, which may be due to the occurrence of apoptosis in Huh 7it-1 cells. In a study conducted by Nasirudeen et al, ${ }^{19}$ it was stated that DENV infection in baby hamster kidney, Huh 7it1 , and Vero cells can induce apoptosis. DENV infection in Huh 7it-1 cells showed cell death activity through the mitochondrial pathway, which resulted in low mitochondrial transmembrane potential in Huh 7it-1 cells. Increased p53 expression was also found in Huh 7it-1 cells infected with DENV.

Our results showed that DENV-2 infection increased the viability of Huh 7it-1 cells; however, the addition of PBMCs although decreased infectivity, still increased the cell damage. This increase in cell damage is due to the condition that PBMCs infected with DENV can produce cytokines. DENV can also infect monocyte-derived macrophage cells ${ }^{22}$ and induce cytokines. ${ }^{23}$ In a study conducted by Chen et $\mathrm{al}^{24}$ regarding pathogen recognition receptors toll-like receptor (TLR)2 and TLR6, the results showed that DENV-infected PBMCs upregulates TLR2 and TLR6. The results of ELISA measurements of IL- 6 and tumor necrosis factor (TNF)- $\alpha$ in PBMCs infected with DENV showed an increase in cytokines, and these cytokines were produced through TLR 2 and TLR6 signaling pathways.

The addition of PBMCs to DENV-infected Huh 7it1 cells decreased infectivity of DENV. It may be due to protection by IFNs, which are produced by monocytes. The addition of PBMCs increased Huh 7it-1 cells viability. It may be due to the increase in IL- 6 and TNF- $\alpha$ production by DENV-infected PBMCs as in the previous study. This study has some potential limitations, such as we could only include one variety of viruses (DENV) and only two varieties of MOI. The result of this study can contribute in knowledge of dengue pathogenesis related to the role of monocytes in liver damage. In conclusion, the addition of PBMCs to Huh 7it-1 cells infected with DENV-2 decreased the infectivity of DENV and increased Huh 7it-1 cells viability.

\section{Conflict of Interest}

The authors affirm no conflict of interest in this study.

\section{Acknowledgment}

We would like to thank subject health volunteer, Dana IImu Pengetahuan Indonesia (DIPI), and Indonesian Endownment Fund for Education (LPDP) for funding this research.

\section{Funding Sources}

This study was funded by Dana IImu Pengetahuan Indonesia (DIPI) and Indonesian Endownment Fund for Education (LPDP).

\section{REFERENCES}

1. Halstead SB, editor. Dengue (tropical medicine: science and practice). London: Imperial College Press; 2008. p. 485.

2. World Health Organization. Global strategy for dengue prevention and control 2012-2020. Switzerland: WHO Library Cataloguing-in-Publication Data; 2012. p. 35.

3. Brady OJ, Gething PW, Bhatt S, Messina JP, Brownstein JS, Hoen $A G$, et al. Refining the global spatial limits of dengue virus transmission by evidence-based consensus. PLoS Negl Trop Dis. 2012;6(8):e1760.

4. Halstead SB, Porterfield JS, O'Rourke EJ. Enhancement of dengue virus infection in monocytes by flavivirus antisera. Am J Trop Med Hyg. 1980;29(4):638-42.

5. Noisakran S, Onlamoon N, Songprakhon P, Hsiao HM, Chokephaibulkit K, Perng GC. Cells in dengue virus infection in vivo. Adv Virol. 2010;2010:164878.

6. Bunyaratvej A, Butthep P, Yoksan S, Bhamarapravati N. Dengue viruses induce cell proliferation and morphological changes of endothelial cells. Southeast Asian J Trop Med Public Health. 1997;28 Suppl 3:32-7. 
7. Seneviratne SL, Malavige GN, de Silva HJ. Pathogenesis of liver involvement during dengue viral infections. Trans R Soc Trop Med Hyg. 2006;100(7):608-14.

8. Marianneau P, Steffan AM, Royer C, Drouet MT, Jaeck D, Kirn A, et al. Infection of primary cultures of human Kupffer cells by dengue virus: no viral progeny synthesis, but cytokine production is evident. J Virol. 1999;73(6):5201-6.

9. Lin YL, Liu CC, Lei HY, Yeh TM, Lin YS, Chen RM, et al. Infection of five human liver cell lines by dengue-2 virus. J Med Virol. 2000;60(4):425-31.

10. Thepparit C, Smith DR. Serotype-specific entry of dengue virus into liver cells: identification of the 37-kilodalton/67-kilodalton high-affinity laminin receptor as a dengue virus serotype 1 receptor. J Virol. 2004;78(22):12647-56.

11. Dewi BE, Takasaki T, Kurane I. Peripheral blood mononuclear cells increase the permeability of dengue virus-infected endothelial cells in association with downregulation of vascular endothelial cadherin. J Gen Virol. 2008;89(Pt 3):642-52.

12. Malkovský M, Loveland B, North M, Asherson GL, Gao L, Ward $P$, et al. Recombinant interleukin-2 directly augments the cytotoxicity of human monocytes. Nature. 1987;325(6101):262-5.

13. Apriyanto DR, Aoki C, Hartati S, Hanafi M, Kardono LB, Arsianti A, et al. Anti-hepatitis C virus activity of a crude extract from Longan (Dimocarpus longan Lour.) leaves. Jpn J Infect Dis. 2016;69(3):213-20.

14. Couvelard A, Marianneau P, Bedel C, Drouet MT, Vachon F, Hénin D, et al. Report of a fatal case of dengue infection with hepatitis: demonstration of dengue antigens in hepatocytes and liver apoptosis. Hum Pathol. 1999;30(9):1106-10.

15. Lin YL, Liu CC, Chuang JI, Lei HY, Yeh TM, Lin YS, et al. Involvement of oxidative stress, NF-IL-6, and RANTES expression in dengue2-virus-infected human liver cells. Virology. 2000;276(1):114-26.
16. Saha AK, Maitra S, Hazra SC. Spectrum of hepatic dysfunction in 2012 dengue epidemic in Kolkata, West Bengal. Indian J Gastroenterol. 2013;32(6):400-3.

17. Lin YL, Lei HY, Lin YS, Yeh TM, Chen SH, Liu HS. Heparin inhibits dengue-2 virus infection of five human liver cell lines. Antiviral Res. 2002;56(1):93-6.

18. Scott RM, Nisalak A, Cheamudon U, Ser idhoranakul S, Nimmannitya S. Isolation of dengue viruses from peripheral blood leukocytes of patients with hemorrhagic fever. J Infect Dis. 1980;141(1):1-6.

19. Nasirudeen AM, Wang L, Liu DX. Induction of p53-dependent and mitochondria-mediated cell death pathway by dengue virus infection of human and animal cells. Microbes Infect. 2008;10(10-11):1124-32.20.

20. Lang J, Vera D, Cheng Y, Tang H. Modeling dengue virus-hepatic cell interactions using human pluripotent stem cell-derived hepatocyte-like cells. Stem Cell Reports. 2016;7(3):341-54.

21. Gresser I, Tovey MG, Bandu MT, Maury C, Brouty-Boye D. Role of interferon in the pathogenesis of virus diseases in mice as demonstrated by the use of anti-interferon serum. I. Rapid evolution of encephalomyocarditis virus infection. J Exp Med. 1976;144(5):1305-15.

22. Kurane I, Kontny U, Janus J, Ennis FA. Dengue-2 virus infection of human mononuclear cell lines and establishment of persistent infections. Arch Virol. 1990;110(1-2):91-101.

23. Bosch I, Xhaja K, Estevez L, Raines G, Melichar H, Warke RV, et al. Increased production of interleukin-8 in primary human monocytes and in human epithelial and endothelial cell lines after dengue virus challenge. J Virol. 2002;76(11):5588-97.

24. Chen J, Ng MM, Chu JJ. Activation of TLR2 and TLR6 by dengue NS1 protein and its implications in the immunopathogenesis of dengue virus infection. PLoS Pathog. 2015;11(7):e1005053. 